\title{
Dissociating Linguistic Processes in the Left Inferior Frontal Cortex with Transcranial Magnetic Stimulation
}

\author{
Patricia M. Gough, ${ }^{1}$ Anna C. Nobre, ${ }^{1}$ and Joseph T. Devlin ${ }^{2}$ \\ ${ }^{1}$ Department of Experimental Psychology and ${ }^{2}$ Center for Functional Magnetic Resonance Imaging of the Brain, University of Oxford, Oxford OX3 9DU, \\ United Kingdom
}

\begin{abstract}
Is the left inferior frontal cortex (LIFC) a single functional region, or can it be subdivided into distinct areas that contribute differently to word processing? Here we used transcranial magnetic stimulation (TMS) to investigate anterior and posterior LIFC when the meaning and sound of words were being processed. Relative to no stimulation, TMS of the anterior LIFC selectively increased response latencies when participants focused on the meaning of simultaneously presented words (i.e., synonym judgments) but not when they focused on the sound pattern of the words (i.e., homophone judgments). In contrast, the opposite dissociation was observed in the posterior LIFC, where stimulation selectively interfered with the phonological but not the semantic task. This double dissociation shows functionally distinct subdivisions of the LIFC that can be understood in terms of separable corticocortical connections linking the anterior LIFC to temporal pole regions associated with semantic memory and the posterior LIFC to temporoparietal regions involved in auditory speech processing.
\end{abstract}

Key words: TMS; language; semantics; phonology; frontotemporal connections; Broca's area

\section{Introduction}

Historically, Broca's area has been viewed as the element of the language circuit responsible for speech production (Broca, 1861; Geschwind, 1967), although more recent studies have revealed its role in multiple aspects of comprehension as well (Zurif et al., 1972; Hagoort et al., 2004). More generally, Broca's area is only part of the larger left inferior frontal cortex (LIFC) region, which is commonly engaged in language paradigms. What is not yet clear is whether the region as a whole is commonly engaged by linguistic tasks or whether different elements of language processing such as syntax, semantics, and phonology engage separate subregions. A consensus has begun to emerge that within the LIFC there is a rostrocaudal division of labor for semantic and phonological processing (Buckner et al., 1995; Fiez, 1997); that is, tasks that focus on the meaning of words (i.e., semantics) engage the anterior and ventral parts of the LIFC, including the pars orbitalis and pars triangularis (Petersen et al., 1989; Demonet et al., 1992; Kapur et al., 1994), whereas those that focus on the sound pattern of words (i.e., phonology) engage more posterior and dorsal LIFC regions, such as the pars opercularis and ventral premotor cortex (Demonet et al., 1992; Zatorre et al., 1992; Rumsey et al., 1997). The actual evidence in support of this claim, however, remains equivocal. Some direct comparisons of seman-

\footnotetext{
Received April 5, 2005; revised July 19, 2005; accepted July 20, 2005.

This work was supported by the Medical Research Council (J.T.D.). We thank Matthew Rushworth, Cathy Price, and two anonymous reviewers for helpful discussions.

Correspondence should be addressed to Dr. Joseph T. Devlin, Center for Functional Magnetic Resonance Imaging of the Brain, Department of Clinical Neurology, John Raddliffe Hospital, University of Oxford, Headley Way, Headington, 0xford 0X3 9DU, UK. E-mail: devlin@fmrib.ox.ac.uk.

D01:10.1523/JNEUROSCI.2307-05.2005

Copyright $\odot 2005$ Society for Neuroscience $\quad 0270-6474 / 05 / 258010-07 \$ 15.00 / 0$
}

tic and phonological processing found significant differences within the LIFC (Poldrack et al., 1999; McDermott et al., 2003; Gitelman et al., 2005), whereas others found differences only at a lenient statistical threshold (Price et al., 1997; Otten and Rugg, 2001; Roskies et al., 2001) or not at all (Demonet et al., 1992). In addition, several recent studies have explicitly shown common LIFC activations (Barde and Thompson-Schill, 2002; Gold and Buckner, 2002; Devlin et al., 2003). These findings offer two possible interpretations. Semantic and phonological tasks may require both anterior and posterior LIFC involvement, but to different extents, or there may be a true rostrocaudal division between semantic and phonological processing that is masked by automatic processing of task-irrelevant information. For example, even if a task requires only semantic information (and thus engages the anterior LIFC), skilled readers will automatically access phonological information as well (engaging the posterior LIFC), although it is not required to perform the task (Van Orden et al., 1988; MacLeod, 1991; Price et al., 1996). Here we used transcranial magnetic stimulation (TMS) to differentiate between these competing hypotheses.

\section{Materials and Methods}

TMS uses a rapidly changing current within a conducting coil to induce a strong, but relatively focal, magnetic field. When placed on the scalp, the magnetic field induces a physiological response (i.e., depolarization, spiking, or both) in the underlying neural tissue (Barker et al., 1985). This introduces transient noise into the neural computation being performed, and when the tissue is required to perform a task, this can lead to longer reaction times (RTs) or even errors (Walsh and Cowey, 2000). For instance, stimulation of the anterior LIFC increased RTs relative to no stimulation when subjects focused on the meaning of a visually presented word (Devlin et al., 2003; Kohler et al., 2004). In contrast, stimulation 
had no effect when subjects attended to visual properties of the word on the screen. Similarly, stimulation of the posterior LIFC selectively increased errors in a phonological, but not a visual, working memory task (Nixon et al., 2004). These results confirm that the LIFC is necessary for semantic and phonological judgments but cannot differentiate between the competing hypotheses regarding LIFC organization because they are predicted by both.

This study tested for a double dissociation of semantic and phonological processing within the LIFC. Participants performed two tasks that involved equivalent stimulus presentation and responses. In both, participants made judgments about pairs of words presented simultaneously; only the nature of the comparison differed. During the semantic task, they decided whether the words meant the same thing (i.e., were synonyms). During the phonological task, they decided whether the words sounded exactly alike (i.e., were homophones). Each participant completed both tasks, with and without stimulation of the anterior and posterior LIFC, in a fully balanced, within-subject, two-by-two-by-two design (task by site by stimulation). If the LIFC exhibited a rostrocaudal division of labor, then this would predict a three-way interaction, during which stimulation of the anterior LIFC interferes with performance on the semantic but not the phonological task, whereas stimulation of the posterior LIFC interferes with performance on the phonological but not the semantic task. However, if both regions of the LIFC were required for the semantic and phonological tasks, then this would predict a main effect of TMS, indicating that stimulation in either region interfered with performance across tasks. To rule out the possibility of nonspecific TMS effects, an additional control task was included that was not expected to be affected by LIFC stimulation. In this task, participants decided whether two meaningless strings of letters were identical.

Participants. Eleven adults (six women, five men; age range, 19-29 years) participated in the main experiment. All were right-handed, native British English speakers without a personal or family history of epilepsy or other neurological conditions. Each gave informed consent after the experimental procedure was explained. Testing sessions lasted $\sim 1.5 \mathrm{~h}$, and subjects were paid for their participation after each session. The experiment was approved by the Local Research Ethics Committee.

Localization procedure. Each volunteer participated in two separate sessions, separated by at least $6 \mathrm{~d}$. Within a session, either the anterior or the posterior LIFC was tested, with the order counterbalanced over participants. A session began when an initial test site was chosen on the basis of either frameless stereotaxy (when available) or scalp measurements.

Four participants had an anatomical magnetic resonance imaging (MRI) scan (Turbo-fast low-angle shot sequence; T1-weighted; $\mathrm{B}_{0}=3 \mathrm{~T}$; repetition time $=15 \mathrm{~ms}$; echo time $=6.9 \mathrm{~ms} ; 1 \times 1 \times 1.5 \mathrm{~mm}$ voxels) that enabled us to use frameless stereotaxy to position the TMS coil on the scalp (Paus et al., 1997). In these, the pars opercularis and pars orbitalis were marked in parasagittal slices of the scan. A Polaris infrared camera (Northern Digital, Waterloo, Ontario, Canada) tracked the subject's head, and BrainSight software (Rogue Research, Montreal, Quebec, Canada) registered the head to the MRI scan. The TMS coil was placed at the approximate site of stimulation, and its position was adjusted until the estimated direction of maximum field intensity intersected the target stimulation site on the MRI scan. This scalp location was then used as the initial testing site.

For subjects who had no MRI scan, the initial site was identified by using the average scalp measurements of the anterior and posterior LIFC, as identified by using frameless stereotaxy in a separate group of volunteers. Nine volunteers (three women, six men) with structural MRI scans who did not participate in the main experiment went through the above localization procedure by means of frameless stereotaxy. Their putative stimulation sites were then measured on the scalp, and the group mean values were calculated. The anterior stimulation site was $2.5 \mathrm{~cm}$ posterior to the canthus along the canther-tragus line and $3 \mathrm{~cm}$ superior to the line; the posterior stimulation site was $4.5 \mathrm{~cm}$ posterior and $6 \mathrm{~cm}$ superior to the line.

For each subject, the coil was placed tangentially to the head at the point of contact and held so that its handle pointed directly downward. A 70-mm-diameter figure-of-eight coil was used with a MagStim RAPID stimulator (Magstim, Whitland, Carmarthenshire, UK). Stimulation was set at $60 \%$ of the stimulator output for all participants, and stimulation always involved three pulses separated by $100 \mathrm{~ms}$, starting $100 \mathrm{~ms}$ after stimulus onset. The TMS train frequency, intensity, and duration were well within internationally established safety limits (Wassermann, 1998).

Functional localization began with one of two "localizer tasks." Localizer tasks were designed to require semantic or phonological processing of the stimuli and were equivalent in stimulus parameters and response requirements. The tasks involved presenting a fixation cross for $1000 \mathrm{~ms}$ immediately followed by two simultaneously presented words above and below the cross for $500 \mathrm{~ms}$. The screen cleared, and participants had 1700 $\mathrm{ms}$ to make a response. For the anterior site, participants decided whether the words referred to items from the same category (e.g., shovelhoe); for the posterior site, participants decided whether the two words rhymed (e.g., coat-vote). Responses were indicated by a button press with the right index and middle fingers. Reaction times and accuracy were measured.

Each localizer task included 30 trials, 10 of which were randomly chosen to include stimulation. If TMS facilitated responses by shortening the median RT for correct responses relative to non-TMS trials, the coil was moved $\sim 1 \mathrm{~cm}$ along the surface of the scalp, and another site was tested. If TMS increased RTs, then the site was tested again to determine whether the effect was consistent. Any slowdown in RTs, even a few milliseconds, was qualitatively distinct from the facilitation effects seen at incorrect sites, and consequently the direction of the effect, rather than its magnitude, was the important criterion. When an increase in RTs occurred twice at the same location, that location was used as the stimulation site in the main experiment. In two participants, we were unable to identify a posterior stimulation site that led to a repeatable increase in RTs, and thus their data were excluded from the analyses of the main experiment.

Main experiment. The main experiment was the same for the two testing sessions and consisted of three alternating tasks: synonym judgments (i.e., semantic task), homophone judgments (i.e., phonological task), and visual matching (i.e., control task), each containing 50 trials. Like the localizer tasks, the main experiment used tasks that selectively targeted semantic or phonological processing. The localizers required an approximate match in either meaning (dog-cat) or sound (eye-fly), whereas the main task required an exact match in meaning (idea-notion) or sound (throne-thrown). To generalize the results beyond specific task demands and stimulus materials, the main experiment differed from the localizer tasks in these two respects.

The presentation parameters were identical to those in the localizer tasks, with a $1000 \mathrm{~ms}$ fixation, $500 \mathrm{~ms}$ stimulus duration, and interstimulus interval of $1700 \mathrm{~ms}$. The experiment was organized in 15 blocks of 10 trials each, with all trials in a block from a single task. A participant might see 10 synonym trials, followed by 10 homophone trials, followed by 10 visual trials before the sequence repeated. In addition, there were two versions of the experiment (A and $B$ ), so that no stimuli were repeated across the two testing sessions. One-half of the participants saw version A during testing of the anterior site and the other half saw version $B$, with the order of testing counterbalanced across subjects. Stimuli were matched across tasks for rated familiarity $\left(t_{(320)}=1.3 ; p>0.1\right)$ (Coltheart, 1981) and log-compressed written word frequencies $\left(t_{(355)}<1\right.$; NS) (Kucera and Francis, 1967; Seidenberg and McClelland, 1989). In general, stimuli in the semantic task were slightly longer than those in the phonological task, with a mean letter length of 5.4 versus $4.8\left(t_{(398)}=3.7\right.$; $p<0.001)$ and a mean syllable length of 1.6 versus $1.3\left(t_{(398)}=5.0 ; p<\right.$ 0.001 ). The complete list of stimuli is present in the appendix (available at www.jneurosci.org as supplemental material). To determine whether the stimuli were sufficiently balanced and that no aspect of the stimulus material chosen could account for the effects of TMS stimulation, a purely behavioral version of the experiment was run with 10 additional participants. Although the participants received no TMS stimulation, the data were analyzed according to whether the trials were assigned to TMS versus no TMS in the main experiment. Reaction times were analyzed with a two-way repeated-measures ANOVA to check whether reaction 
times were affected by the TMS or task (semantic or phonological) factors. There was no significant main effect of TMS $\left(F_{(1,9)}<1\right.$; NS), nor was there a significant interaction $\left(F_{(1,9)}=0.0 ; \mathrm{NS}\right)$, indicating that stimulus factors could not account for the TMS findings. The main effect of task, however, approached significance $\left(F_{(1,9)}=5.0 ; p=0.053\right)$, indicating that RTs in the semantic task (mean, $1104 \mathrm{~ms}$ ) were longer than those in the phonological task (mean, $1049 \mathrm{~ms}$ ), which is similar to that seen in the main experiment (see Fig. 2B). Task differences in overall RTs were removed from the main experiment by normalizing the reaction times and measuring TMS effects as percentage change relative to baseline.

In the main experiment, repetitive TMS was delivered pseudorandomly on $40 \%$ of all trials with the same stimulation parameters that were used in the localizer tasks $(10 \mathrm{~Hz} ; 300 \mathrm{~ms}$ duration; $60 \%$ maximum stimulator output). The sequence was constrained to guarantee equal numbers of TMS trials across conditions and also to ensure that no more than two TMS trials occurred sequentially. We did not include sham TMS trials as an additional control condition; instead, the visual matching task served as the control for the other two tasks. As with the localizer tasks, the order of stimulation sites was fully counterbalanced across participants.

Response times were recorded from the onset of the stimuli, and trials with RTs beyond 2 SDs from the mean were excluded [ 114 of 3000 trials $(3.8 \%)]$. In addition, four semantic trials were removed because participants responded at chance (50\%). Median RTs for correct responses per condition per subject were normalized to reflect relative changes caused by stimulation (Schluter et al., 1999; Devlin et al., 2003; Kohler et al., 2004) as follows: $\%$ change $=(\mathrm{RT}$ with TMS $-\mathrm{RT}$ without TMS $) /(\mathrm{RT}$ without TMS) $\times 100$.

These data were then analyzed with a repeated-measures ANOVA, and the interaction was further characterized by paired $t$ tests to assess the significance of site-specific effects. To verify the reliability of the effects in the case of possible violations of the assumptions of the general linear model, these data were also analyzed with nonparametric Wilcoxon signed rank tests, which confirmed the results of the parametric tests.

\section{Results}

The experiment consisted of two stages. Testing began by using localizer tasks to identify functionally the anterior and posterior stimulation sites. For the anterior site, participants decided whether two simultaneously presented words came from the same category (e.g., potato-turnip) and indicated their response with a key press. For the posterior site, participants decided whether the words rhymed (e.g., vein-pane). The results of the localizer tasks are shown in Figure 1. Stimulation at the anterior testing site increased response latencies in all 11 participants relative to no stimulation, with an average RT increase of $41 \mathrm{~ms}$ (paired $t$ test; $t_{(10)}=3.3 ; p<0.01$ ). The fact that TMS led to a significant slowing of response was not surprising, given that this was the criterion used to identify the stimulation site. What is important, however, was the fact that such a site could be identified in all subjects. A similar pattern was observed at the posterior testing site, where stimulation led to RT increases in 9 of 11 participants, with a mean increase of $52 \mathrm{~ms}\left(t_{(9)}=3.1 ; p<0.05\right)$. In two cases, however, posterior stimulation did not yield reproducible increases in RTs; a potential site was identified as showing an RT increase during initial testing, but subsequent tests at the same location showed decreased RTs. One subject showed a 27 ms slowdown initially and then an $80 \mathrm{~ms}$ facilitation during retesting, whereas the second subject showed a $16 \mathrm{~ms}$ inhibition followed by a $9 \mathrm{~ms}$ facilitation (Fig. 1, gray lines). Both individuals were excluded from the main experiment. At the final test site, TMS produced both qualitatively and quantitatively different results from those of the adjacent stimulated regions (i.e., sites within $2 \mathrm{~cm}$ of the final testing site), where TMS produced a mean

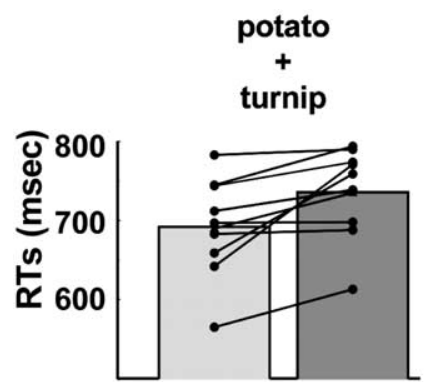

No TMS

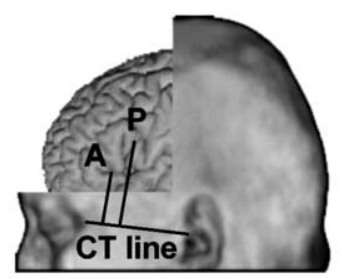

vein

pane

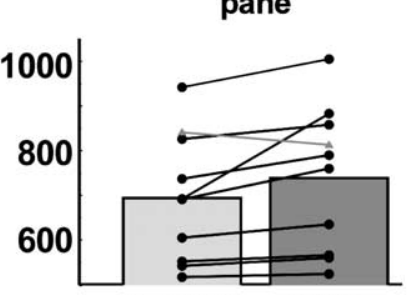

No TMS

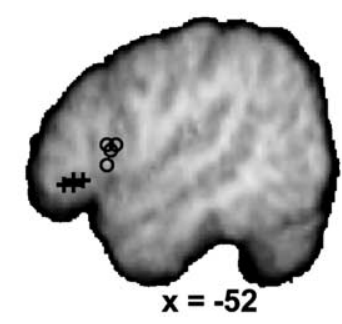

Figure 1. Results of functional localization. The bar plots illustrate group mean RTs for the category judgment task used to localize the anterior stimulation site (top left) and the rhyme judgment tasks used to localize the posterior stimulation site (top right). In both regions, stimulation (dark gray) significantly increased RTs relative to trials without TMS (light gray), but more importantly, a consistent increase in RTs was observed in both locations for 9 of 11 individuals (dark lines). Two subjects (light lines) were excluded from the main experiment for not showing a consistent TMS-induced RT increase in the posterior localization task. Bottom left, Average scalp measurements relative to the canther-tragus (CT) line for the anterior (A) and posterior (P) sites. Bottom right, The same sites on a parasagittal slice through the mean structural image of the four participants with MRI scans. Anterior locations for each individual are marked with crosses; posterior locations are marked with circles. Scale bar, $10 \mathrm{~mm}$.

decrease in RTs of $31 \mathrm{~ms}$, consistent with intersensory facilitation (Walsh and Rushworth, 1999).

The location of the test sites was measured in two ways. First, a scalp measurement was made for each subject by drawing a reference line connecting the canthus of the left eye to the intertragal notch of the ear (Fig. 1C). Two line segments were drawn at right angles to the reference line connecting it to the anterior and posterior sites. On average, the mean anterior position was $2 \mathrm{~cm}$ along the canther-tragus line and $3 \mathrm{~cm}$ superior to it along a perpendicular line. The mean posterior site was $4 \mathrm{~cm}$ posterior and $5.75 \mathrm{~cm}$ superior to the line; therefore, the distance between the two sites was $\sim 3.5 \mathrm{~cm}$ on the surface of the scalp but varied from 1.8 to $4.8 \mathrm{~cm}$ across the nine participants. A second measurement was made in four of the participants who had highresolution structural MRI scans (Fig. 1D). Clear anatomical fiducial marks permitted the use of frameless stereotaxy to identify the cortical sites of stimulation (Paus et al., 1997). In each individual, the anterior site fell on the horizontal ramus of the Sylvian fissure separating the pars triangularis from the pars orbitalis, whereas the posterior site was consistently located just anterior to the ventral limb of the precentral sulcus on the convexity of the pars opercularis. When these locations were transformed into the standard reference space defined by the Montreal Neurological Institute mean brain (MNI152), the average coordinates of the anterior and posterior testing sites were $-52,34$, $-6 \mathrm{~mm}$ and $-52,16,8 \mathrm{~mm}$, respectively. The distances within the four subjects were 1.7, 2.6, 2.7 and $3.0 \mathrm{~cm}$ apart (an average of $2.5 \mathrm{~cm}$ ) on the cortical surface. Previous studies suggest that TMS 


\section{A. Accuracy}
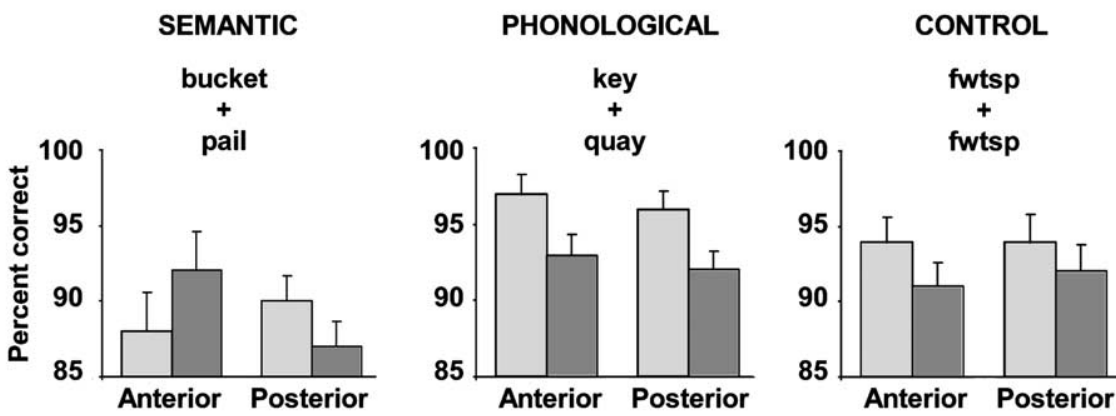

B. Normalized reaction times

SEMANTIC

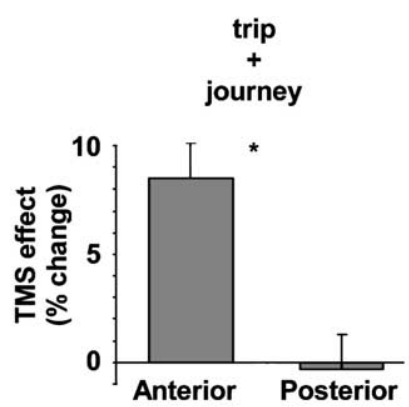

PHONOLOGICAL
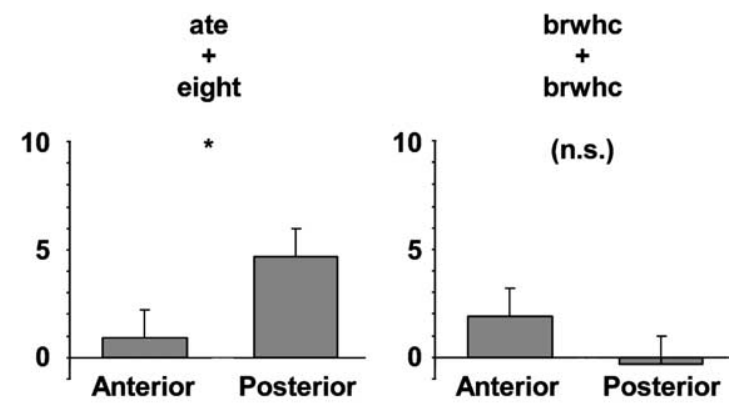

C. Reaction times

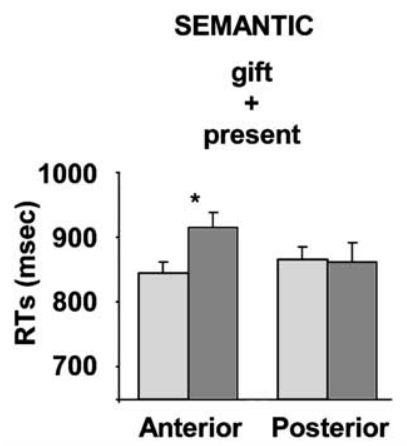

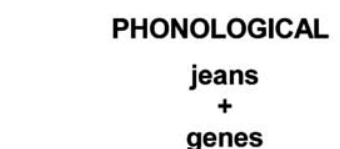

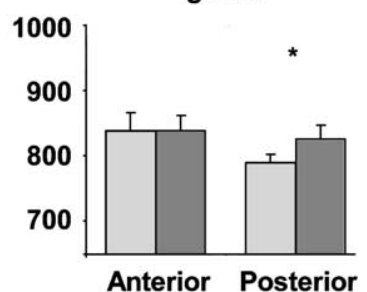

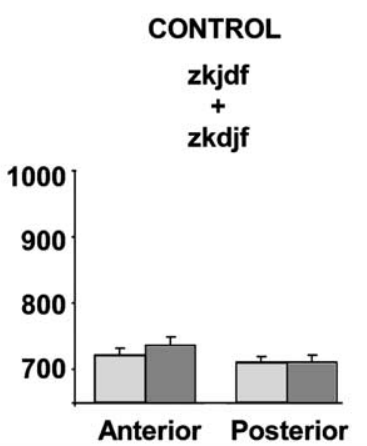

Figure 2. Effects of anterior and posterior stimulation on performance during synonym judgments (left), homophone judgments (middle), and visual matching (right). $\boldsymbol{A}$, Accuracy measures across tasks and conditions. $\boldsymbol{B}$, Normalized TMS effects expressed as a change relative to the non-TMS baseline trials. $C$, Group mean reaction times per condition. Trials without TMS are shown in light gray; those with TMS are shown in dark gray. Error bars indicate SEM adjusted to reflect the between-condition variance used in repeated-measure designs (Loftus and Masson, 1994).

has a functional resolution of $\sim 1 \mathrm{~cm}$ (Brasil-Neto et al., 1992; Pascual-Leone et al., 1999; Walsh and Rushworth, 1999), consistent with our finding that distinct behavioral effects were detectable when sites $\sim 3.5 \mathrm{~cm}$ apart on the surface of the scalp or $\sim 2.5$ $\mathrm{cm}$ apart on the cortical surface were stimulated.

The behavioral results are summarized in Figure 2. Accuracy data were analyzed with a two-by-two-by-two repeatedmeasures ANOVA. The independent factors were task (semantic and phonological), site (anterior and posterior), and stimulation (yes or no). There was a significant main effect of task $\left(F_{(1,8)}=\right.$ 7.7; $p<0.05)$, indicating that participants responded less accurately for synonym trials $(89.8 \%)$ than for homophone trials (93.9\%), but no other main effects or interactions reached signif- icance. TMS did not affect performance in either task when measured in terms of accuracy.

To characterize the effects of TMS on RTs, the RTs of each participant were normalized relative to their non-TMS response times (baseline), expressed as percentage change from baseline (Schluter et al., 1999; Devlin et al., 2003; Kohler et al., 2004), and then analyzed with a two-bytwo repeated-measures ANOVA. The independent factors were task (semantic and phonological) and site (anterior and posterior). There were no significant main effects (both $F$ values $<1.5$; NS), but there was a highly significant interaction $\left(F_{(1,8)}\right.$ $=13.2 ; p<0.01$ ), indicating that TMS affected the semantic and phonological tasks differently, depending on the stimulation site. Post hoc $t$ tests confirmed that anterior stimulation selectively increased RTs in the semantic $\left(t_{(8)}=3.2 ; p<0.01\right)$ but not the phonological task, whereas posterior stimulation selectively increased RTs in the phonological $\left(_{(8)}=2.2 ; p<\right.$ 0.05 ) but not the semantic task. In the anterior site, stimulation increased RTs in the synonym task by $9 \%$ of the no-TMS baseline, an average of $70 \mathrm{~ms}$. In contrast, there was no significant increase (1\% change) in the homophone task. The opposite pattern was observed in the posterior site, where stimulation increased response times in the homophone task by $5 \%$ (36 ms) but not in the synonym task ( $0 \%$ change; $-4 \mathrm{~ms}$ ). Anterior stimulation selectively interfered with performance on the semantic task, whereas posterior stimulation selectively interfered with the phonological task. As expected, stimulation did not significantly affect performance on the control task. Anteriorally, there was a $2 \%(16 \mathrm{~ms})$ increase in RTs, whereas posteriorly there was no change in RTs $(0 \% ;-1 \mathrm{~ms})$; neither was significant (both $t_{(8)}<1.0$; NS).

Visual inspection of the raw RTs (Fig. 2, bottom row) reveals that the posterior TMS effect for homophones may have been caused by faster responses in the nonTMS baseline rather than a stimulation-induced slowdown. A direct comparison of anterior and posterior non-TMS RTs, however, reveals that the difference was not significant (paired $t$ test; $\left.t_{(8)}=1.7 ; p=0.12\right)$. In fact, faster reaction times, $21 \mathrm{~ms}$ on average, were seen in all three tasks when tested at the posterior relative to the anterior stimulation site. Although this effect was not statistically significant $\left(F_{(1,8)}=1.0 ; p=0.34\right)$, it is consistent with the non-TMS baseline being faster for posterior than for anterior stimulation. This difference cannot be attributed to stimuli or task differences across stimulation sites, because both were counterbalanced across subjects; instead, it may relate to the subjective experience of posterior and anterior LIFC stimulation. Stimulation at the more anterior site is more uncomfortable be- 
cause of increased peripheral nerve stimulation and thus may be responsible for a generalized slowing of responses. We suggest that slowing above baseline performance in three of the four phonological conditions may have resulted from different factors. For the anterior site, both the baseline and TMS conditions were slower because of increased discomfort, although this was not a significant effect. In contrast, the posterior stimulation condition was slower because of TMS stimulation. This explanation is consistent with the TMS-induced slowdown for rhyme judgments in the posterior localizer task and the selective disruption of phonological working memory reported previously by Nixon et al. (2004).

\section{Discussion}

Stimulation of the anterior LIFC increased response latencies relative to no stimulation in the synonym but not the homophone task, whereas the opposite pattern was observed for stimulation of the posterior LIFC. We interpret these findings in terms of a regionally specific disruption of semantic or phonological processing. However, the semantic task was more difficult for the participants than the phonological task, as indexed by both increased error rates and longer RTs. Consequently, it is possible that relative to the phonological task, synonym judgments required additional cognitive resources. For instance, homophone decisions are unambiguous: two words either sound identical or do not. In contrast, no two words are exact synonyms; therefore, the task may have required additional processing to resolve this ambiguity. Previous imaging studies have shown that the anterior LIFC is engaged in resolving underconstrained tasks (Gold and Buckner, 2002; Gold et al., 2005), and thus stimulation of this region could have disrupted these nonsemantic processes. By itself, we find this explanation unlikely, because it cannot explain why stimulation of the anterior LIFC selectively affected the highly constrained semantic decision tasks used by Devlin et al. (2003) or Kohler et al. (2004). However, a combination of semantic and nonsemantic factors may have exaggerated the TMS effect seen with anterior stimulation in our experiment, offering a potential explanation for why the anterior TMS effect was both larger and more robust than the posterior effect.

Our results show a rostrocaudal division of labor in the LIFC that has been suggested but not shown in previous TMS studies (Devlin et al., 2003; Kohler et al., 2004; Nixon et al., 2004). This is consistent with imaging studies that show preferential engagement of these areas across various semantic and phonological tasks (Buckner et al., 1995; Poldrack et al., 1999; Bokde et al., 2001; Otten and Rugg, 2001; Devlin et al., 2003; McDermott et al., 2003; Gitelman et al., 2005) but inconsistent with the hypothesis that semantic and phonological tasks each rely on both the anterior and the posterior LIFC. Those studies that have reported either no differences (Demonet et al., 1992; Price et al., 1997) or common activation (Barde and Thompson-Schill, 2002; Gold and Buckner, 2002; Devlin et al., 2003) in the LIFC for semantic and phonological processing therefore may reflect the fact that words automatically engage semantic and phonological processing even when not required to perform the task (Van Orden et al., 1988; MacLeod, 1991; Price et al., 1996). The use of TMS in this study has allowed us to separate regions necessary for performance in each task from those that are involved only incidentally and has revealed an anteroposterior division within the LIFC for semantic and phonological processing of words.

We hypothesize that this functional double dissociation may be caused by separate corticocortical pathways linking specific prefrontal areas to distinct temporal lobe regions. In macaques, the uncinate fasciculus links perirhinal, entorhinal, and anterolateral superior temporal regions with ventral prefrontal areas, including lateral area 47/12 (Petrides and Pandya, 2002a,b). In contrast, the superior longitudinal fasciculus connects the temporoparietal junction with areas 44 and F5 (i.e., ventral premotor cortex) (Petrides and Pandya, 1984; Cipolloni and Pandya, 1999). Moreover, short-association fibers (U-fibers) link areas 44-45 and 45-47/12. Petrides and Pandya (1994, 2002a,b) have shown that areas 44,45 , and $47 / 12$ are cytoarchitectonic homologs of Brodmann areas 44, 45, and 47 in humans, which correspond to partes opercularis, triangularis, and orbitalis, respectively (Amunts et al., 1999; Tomaiuolo et al., 1999). If the macaque pattern of connectivity is preserved in humans, then the anterior LIFC (i.e., partes orbitalis and triangularis) has reciprocal connections with anterior temporal regions associated with semantic memory (Hodges et al., 1995; Nobre and McCarthy, 1995; Vandenberghe et al., 1996; Devlin et al., 2002). The posterior LIFC (i.e., pars opercularis and ventral premotor cortex), in contrast, has reciprocal connections with the posterior supratemporal areas that are generally involved in auditory processing (Griffiths and Warren, 2002) but also specifically associated with speech perception (Hickok and Poeppel, 2000; Giraud and Price, 2001; Wise et al., 2001; Scott and Johnsrude, 2003). A final set of reciprocal connections links the anterior and posterior LIFC areas, providing an anatomical substrate for integrating semantic and phonological information.

These pathways are consistent with claims that the anterior LIFC may operate as part of a semantic executive system (Wagner et al., 2001; Gold et al., 2005), whereas the posterior LIFC plays a crucial role in integrating acoustic (sensory) information with articulatory (motor) patterns (Wise et al., 2001; Scott and Johnsrude, 2003). Patients with LIFC lesions do not typically have semantic memory deficits, although their ability to retrieve specific semantic information, select among multiple options, or inhibit competing alternatives can be impaired (Costello and Warrington, 1989; Robinson et al., 1998; Thompson-Schill et al., 1998). Similarly, LIFC lesions can impair phonological processing (Gow and Caplan, 1996), although this is more generally associated with posterior supratemporal lobe lesions (Bogen and Bogen, 1976). Presumably, both posterior LIFC and supratemporal plane lesions disrupt the sensorimotor integration necessary for phonological processing (Wise et al., 2001; Fadiga et al., 2002; Scott and Johnsrude, 2003; Watkins et al., 2003; Watkins and Paus, 2004). The LIFC provides the substrate for integrating parallel linguistic streams, including semantic, phonological, and presumably syntactic information, to resolve ambiguities via the constraints provided by each type of information (Jackendoff, 2002; Hagoort, 2003). These results help to define the LIFC as a functionally and anatomically heterogeneous zone that plays a crucial role in the integration and modulation of abstract (i.e., supramodal) linguistic information.

\section{References}

Amunts K, Schleicher A, Burgel U, Mohlberg H, Uylings HBM, Zilles K (1999) Broca's region revisited: cytoarchitecture and intersubject variability. J Comp Neurol 412:319-341.

Barde LH, Thompson-Schill SL (2002) Models of functional organization of the lateral prefrontal cortex in verbal working memory: evidence in favor of the process model. J Cogn Neurosci 14:1054-1063.

Barker AT, Jalinous R, Freeston IL (1985) Non-invasive magnetic stimulation of human motor cortex. Lancet 1:1106-1107. 
Bogen JE, Bogen GM (1976) Wernicke's region: where is it? Ann NY Acad Sci 280:834-843.

Bokde AL, Tagamets MA, Friedman RB, Horwitz B (2001) Functional interactions of the inferior frontal cortex during the processing of words and word-like stimuli. Neuron 30:609-617.

Brasil-Neto JP, McShane LM, Fuhr P, Hallet M, Cohen LG (1992) Topographic mapping of the human motor cortex with magnetic stimulation: factors affecting accuracy and reproducibility. J Electroencephalogr Clin Neurophysiol 85:9-16.

Broca P (1861) Remarques sur le siège de la faculté du langage articulé; suivies d'une observation d'aphemie. Bull Soc Anatomique Paris 6:330-357.

Buckner RL, Raichle ME, Petersen SE (1995) Dissociation of human prefrontal cortical areas across different speech production tasks and gender groups. J Neurophysiol 74:2163-2173.

Cipolloni PB, Pandya DN (1999) Cortical connections of the frontoparietal opercular areas in the rhesus monkey. J Comp Neurol 403:431-458.

Coltheart M (1981) The MRC psycholinguistics database. Q J Exp Psychol 33A:497-505.

Costello AL, Warrington EK (1989) Dynamic aphasia: the selective impairment of verbal planning. Cortex 25:103-114.

Demonet J-F, Chollet F, Ramsay S, Cardebat D, Nespoulous J-L, Wise R, Rascol A, Frackowiak R (1992) The anatomy of phonological and semantic processing in normal subjects. Brain 115:1753-1768.

Devlin JT, Moore CJ, Mummery CJ, Gorno-Tempini ML, Phillips JA, Noppeney U, Frackowiak RS, Friston KJ, Price CJ (2002) Anatomic constraints on cognitive theories of category specificity. NeuroImage 15:675-685.

Devlin JT, Matthews PM, Rushworth MF (2003) Semantic processing in the left inferior prefrontal cortex: a combined functional magnetic resonance imaging and transcranial magnetic stimulation study. J Cogn Neurosci 15:71-84.

Fadiga L, Craighero L, Buccino G, Rizzolatti G (2002) Speech listening specifically modulates the excitability of tongue muscles: a TMS study. Eur J Neurosci 15:399-402.

Fiez JA (1997) Phonology, semantics, and the role of the left inferior prefrontal cortex. Hum Brain Mapp 5:79-83.

Geschwind N (1967) The varieties of naming errors. Cortex 3:97-112.

Giraud AL, Price CJ (2001) The constraints functional neuroimaging places on classical models of word processing. J Cogn Neurosci 13:1-12.

Gitelman DR, Nobre AC, Sonty S, Parrish TB, Mesulam MM (2005) Language network specializations: an analysis with parallel task designs and functional magnetic resonance imaging. NeuroImage 26:975-985.

Gold BT, Buckner RL (2002) Common prefrontal regions coactivate with dissociable posterior regions during controlled semantic and phonological tasks. Neuron 35:803-812.

Gold BT, Balota DA, Kirchhoff BA, Buckner RL (2005) Common and dissociable activation patterns associated with controlled semantic and phonological processing: evidence from fMRI adaptation. Cereb Cortex 15:1438-1450.

Gow Jr DW, Caplan D (1996) An examination of impaired acoustic-phonetic processing in aphasia. Brain Lang 52:386-407.

Griffiths TD, Warren JD (2002) The planum temporale as a computational hub. Trends Neurosci 25:348-353.

Hagoort P (2003) How the brain solves the binding problem for language: a neurocomputational model of syntactic processing. NeuroImage 20 [Suppl 1]:18-29.

Hagoort P, Hald L, Bastiaansen M, Petersson KM (2004) Integration of word meaning and world knowledge in language comprehension. Science 304:438-441.

Hickok G, Poeppel D (2000) Towards a functional neuroanatomy of speech perception. Trends Cogn Sci 4:131-138.

Hodges JR, Graham N, Patteron K (1995) Charting the progression in semantic dementia: implications for the organization of semantic memory. Memory 3:463-495.

Jackendoff RS (2002) Foundations of language: brain, meaning, grammar, evolution. Oxford: Oxford UP.

Kapur S, Rose R, Liddle PF, Zipursky RB, Brown GM, Stuss D, Houle S, Tulving E (1994) The role of the left prefrontal cortex in verbal processing: semantic processing or willed action? NeuroReport 5:2193-2196.

Kohler S, Paus T, Buckner RL, Milner B (2004) Effects of left inferior pre- frontal stimulation on episodic memory formation: a two-stage fMRIrTMS study. J Cogn Neurosci 16:178-188.

Kucera H, Francis WN (1967) Computational analysis of present-day American English. Providence, RI: Brown UP.

Loftus GR, Masson MEJ (1994) Using confidence-intervals in withinsubject designs. Psychon Bull Rev 1:476-490.

MacLeod CM (1991) Half a century of research on the Stroop effect: an integrative review. Psychol Bull 109:163-203.

McDermott KB, Petersen SE, Watson JM, Ojemann JG (2003) A procedure for identifying regions preferentially activated by attention to semantic and phonological relations using functional magnetic resonance imaging. Neuropsychologia 41:293-303.

Nixon P, Lazarova J, Hodinott-Hill I, Gough P, Passingham R (2004) The inferior frontal gyrus and phonological processing: an investigation using rTMS. J Cogn Neurosci 16:289-300.

Nobre AC, McCarthy G (1995) Language-related field potentials in the anterior-medial temporal lobe. II. Effects of word type and semantic priming. J Neurosci 15:1090-1098.

Otten LJ, Rugg MD (2001) Task-dependency of the neural correlates of episodic encoding as measured by fMRI. Cereb Cortex 11:1150-1160.

Pascual-Leone A, Bartres-Faz D, Keenan JP (1999) Transcranial magnetic stimulation: studying the brain-behaviour relationship by induction of "virtual lesions." Philos Trans R Soc Lond B Biol Sci 354:1229-1238.

Paus T, Jech R, Thompson CJ, Comeau R, Peters T, Evans AC (1997) Transcranial magnetic stimulation during positron emission tomography: a new method for studying connectivity of the human cerebral cortex. J Neurosci 17:3178-3184.

Petersen SE, Fox PT, Posner MI, Mintun M, Raichle ME (1989) Positron emission tomographic studies of the processing of single words. J Cogn Neurosci 1:153-170.

Petrides M, Pandya DN (1984) Projections to the frontal cortex from the posterior parietal region in the rhesus monkey. J Comp Neurol 228:105-116.

Petrides M, Pandya DN (1994) Comparative architectonic analysis of the human and the macaque frontal cortex. In: Handbook of neuropsychology (Boller F, Grafman J, eds), pp 17-58. Philadelphia: Elsevier.

Petrides M, Pandya DN (2002a) Comparative cytoarchitectonic analysis of the human and the macaque ventrolateral prefrontal cortex and corticocortical connection patterns in the monkey. Eur J Neurosci 16:291310.

Petrides M, Pandya DN (2002b) Association pathways of the prefrontal cortex and functional observations. In: Principles of frontal lobe function (Stuss DT, Knight RT, eds), pp 31-50. Oxford: Oxford UP.

Poldrack RA, Wagner AD, Prull MW, Desmond JE, Glover GH, Gabrieli JD (1999) Functional specialization for semantic and phonological processing in the left inferior prefrontal cortex. NeuroImage 10:15-35.

Price CJ, Wise RJS, Frackowiak RSJ (1996) Demonstrating the implicit processing of visually presented words and pseudowords. Cereb Cortex 6:62-70.

Price CJ, Moore CJ, Humphreys GW, Wise RJ (1997) Segregating semantic from phonological processes during reading. J Cogn Neurosci 9:727-733.

Robinson G, Blair J, Cipolotti L (1998) Dynamic aphasia: an inability to select between competing verbal responses? Brain 121:77-89.

Roskies AL, Fiez JA, Balota DA, Raichle MA, Petersen SE (2001) Taskdependent modulation of regions in the left inferior frontal cortex during semantic processing. J Cogn Neurosci 13:829-843.

Rumsey JM, Horwitz B, Donahue BC, Nace K, Maisog JM, Andreason P (1997) Phonological and orthographic components of word recognition: a PET-rCBF study. Brain 120:739-759.

Schluter ND, Rushworth MFS, Mills KR, Passingham RE (1999) Signal-, set-, and movement-related activity in the human premotor cortex. Neuropsychologia 37:233-243.

Scott SK, Johnsrude IS (2003) The neuroanatomical and functional organization of speech perception. Trends Neurosci 26:100-107.

Seidenberg MS, McClelland JL (1989) A distributed, developmental model of word recognition and naming. Psychol Rev 96:523-568.

Thompson-Schill SL, Swick D, Farah MJ, D’Esposito M, Kan IP, Knight RT (1998) Verb generation in patients with focal frontal lesions: a neuropsychological test of neuroimaging findings. Proc Natl Acad Sci USA 95:15855-15860.

Tomaiuolo F, MacDonald JD, Caramanos Z, Posner G, Chiavaras M, Evans AC, Petrides M (1999) Morphology, morphometry and probability 
mapping of the pars opercularis of the frontal gyrus: an in vivo MRI analysis. Eur J Neurosci 11:3033-3046.

Vandenberghe R, Price CJ, Wise R, Josephs O, Frackowiak RSJ (1996) Functional anatomy of a common semantic system for words and pictures. Nature 383:254-256.

Van Orden GC, Johnson JC, Hale BL (1988) Word identification in reading proceeds from spelling to sound to meaning. J Exp Psychol Learn Mem Cogn 14:371-386.

Wagner AD, Pare-Blagoev EJ, Clark J, Poldrack RA (2001) Recovering meaning: left prefrontal cortex guides controlled semantic retrieval. Neuron 31:329-338.

Walsh V, Cowey A (2000) Transcranial magnetic stimulation and cognitive neuroscience. Nat Rev Neurosci 1:73-79.

Walsh V, Rushworth MFS (1999) The use of transcranial magnetic stimulation in neuropsychological testing. Neuropsychologia 37:125-135.

Wassermann EM (1998) Risk and safety of repetitive transcranial mag- netic stimulation: report and suggested guidelines from the International Workshop on the Safety of Repetitive Transcranial Magnetic Stimulation, June 5-7, 1996. Electroencephalogr Clin Neurophysiol 108:1-16.

Watkins K, Paus T (2004) Modulation of motor excitability during speech perception: the role of Broca's area. J Cogn Neurosci 16:978-987.

Watkins KE, Strafella AP, Paus T (2003) Seeing and hearing speech excites the motor system involved in speech production. Neuropsychologia 41:989-994.

Wise RJ, Scott SK, Blank SC, Mummery CJ, Murphy K, Warburton EA (2001) Separate neural subsystems within "Wernicke's area." Brain 124:83-95.

Zatorre RJ, Evans AC, Meyer E, Gjedde A (1992) Lateralization of phonetic and pitch discrimination in speech processing. Science 256:846-849.

Zurif EB, Caramazza A, Myerson R (1972) Grammatical judgments of agrammatic aphasics. Neuropsychologia 10:405-417. 